\title{
Experiments and analyses of upstream-advancing solitary waves generated by moving disturbances
}

\author{
By SEUNG-JOON LEE†, GEORGE T. YATES \\ AND T. YAOTSU WU \\ Engineering Science, California Institute of Technology, \\ Pasadena, CA 91125, USA
}

(Received 25 March 1988 and in revised form 8 July 1988)

In this joint theoretical, numerical and experimental study, we investigate the phenomenon of forced generation of nonlinear waves by disturbances moving steadily with a transcritical velocity through a layer of shallow water. The plane motion considered here is modelled by the generalized Boussinesq equations and the forced Korteweg-de Vries ( $\mathrm{fKdV}$ ) equation, both of which admit two types of forcing agencies in the form of an external surface pressure and a bottom topography. Numerical results are obtained using both theoretical models for the two types of forcings. These results illustrate that within a transcritical speed range, a succession of solitary waves are generated, periodically and indefinitely, to form a procession advancing upstream of the disturbance, while a train of weakly nonlinear and weakly dispersive waves develops downstream of an ever elongating stretch of a uniformly depressed water surface immediately behind the disturbance. This is a beautiful example showing that the response of a dynamic system to steady forcing need not asymptotically tend to a steady state, but can be conspicuously periodic, after an impulsive start, when the system is being forced at resonance.

A series of laboratory experiments was conducted with a cambered bottom topography impulsively started from rest to a constant transcritical velocity $U$, the corresponding depth Froude number $F=U /\left(g h_{0}\right)^{\frac{1}{2}}(g$ being the gravitational constant and $h_{0}$ the original uniform water depth) being nearly the critical value of unity. For the two types of forcing, the generalized Boussinesq model indicates that the surface pressure can be more effective in generating the precursor solitary waves than the submerged topography of the same normalized spatial distribution. However, according to the fKdV model, these two types of forcing are entirely equivalent. Besides these and some other rather refined differences, a broad agreement is found between theory and experiment, both in respect of the amplitudes and phases of the waves generated, when the speed is nearly critical $(0.9<F<1.1)$ and when the forcing is sufficiently weak (the topography-height to water-depth ratio less than 0.15 ) to avoid breaking. Experimentally, wave breaking was observed to occur in the precursor solitary waves at low supercritical speeds (about $1.1<F<1.2$ ) and in the first few trailing waves at high subcritical speeds (about $0.8<F<0.9$ ), when sufficiently forced. For still lower subcritical speeds, the trailing waves behaved more like sinusoidal waves as found in the classical case and the forward-running solitary waves, while still experimentally discernible and numerically predicted for $\mathbf{0 . 6}>$ $F>0.2$, finally disappear at $F \approx 0.2$. In the other direction, as the Froude number is increased beyond $F \approx 1.2$, the precursor soliton phenomenon was found also to

$\dagger$ Current address: Chungnam National University, Dept. Naval Architecture and Ocean Engineering, Daeduk Science Town 300-31 Korea. 
evanesce as no finite-amplitude solitary waves can outrun, nor can any twodimensional waves continue to follow, the rapidly moving disturbance. In this supercritical range and for asymptotically large times, all the effects remain only local to the disturbance. Thus, the criterion of the fascinating phenomenon of the generation of precursor solitons is ascertained.

\section{Introduction}

Since the term soliton was coined by Zabusky \& Kruskal (1965), research exploration of various nonlinear wave phenomena has brought forth a quite dramatic rise in scientific activities in this rapidly developing field. These studies have added important new concepts and understanding to the general subject opened by the first recorded observation of a 'great solitary wave' in 1834 by John Scott Russell and then firmly founded on theoretical basis by Boussinesq, Rayleigh and Korteweg \& de Vries. The impressive conceptual growth and the colourful developments of powerful mathematical methods achieved along the wide frontiers of this field extend far and deep into fluid mechanical, physical, chemical, biological and mathematical disciplines and genuinely constitute an outstanding interdisciplinary subject of modern day mathematical physics. These notable advances can primarily be attributed to the outstanding feature that the model systems possess infinitely many conservation laws rendering the basic equations integrable. Under this premise, methods of solution have been developed, including Bäcklund transforms, inverse scattering and other inverse methods, modulation theory, etc. (recent reviews can be found in Miles 1980; Dodd et al. 1982; Bullough \& Caudrey 1980; Rebbi \& Soliani 1984).

Of particular interest here, we note that these activities have been confined largely to physically closed systems, admitting no exchanges of mass, momentum and energy with external agencies. Furthermore, only limited efforts have been extended to considering problems in more than one horizontal spatial dimension. In this work, we carry out a joint theoretical, numerical and experimental study of a physically open, soliton-bearing system when the system is being continuously forced near resonance, complementing the recent study by $\mathrm{Wu}$ (1987), who also gave a brief survey of this subject.

To facilitate studies of nonlinear dispersive wave phenomena occurring in physically open systems, $\mathrm{Wu}(1979,1981)$ generalized the Boussinesq equations to incorporate possible exchanges of mass, momentum and energy, in more than one space dimension, with some external agencies, and obtained a model for weakly nonlinear and weakly dispersive long waves in water with depth gradually varying in two horizontal dimensions and with moving surface pressure and submerged topography admitted as forcing functions. The two equations for the free-surface elevation and the layer-mean velocity potential are derived using the basic set of transport theorems and the nonlinear boundary conditions. They are called the generalized Boussinesq ( $\mathrm{g}-\mathrm{B}$ ) equations. From their solutions and the expansion equations used therein, the pressure and velocity fields are determined. This model was employed by $\mathrm{Wu} \& \mathrm{Wu}(\mathbf{1 9 8 2 )}$ to give the first reported discovery of the phenomenon of precursor solitons, or the so-called 'runaway solitions'. It has also been applied by Schember (1982) to calculate some three-dimensional nonlinear longwave problems and by Lepelletier (1981) and Lepelletier \& Raichlen (1987) to evaluate several tsunami and harbour oscillation problems. 
A different approach was proposed by Green \& Naghdi $(1976 a, b)$ based on the concept of a directed-sheet model. With the horizontal velocity component assumed independent of the vertical $z$-coordinate and the vertical velocity component varying linearly in $z$, the equations of motion are derived by invoking conservation of mass and momentum in a depth-integrated form together with the exact boundary conditions. Thus, the motion described by the Green-Naghdi $(\mathrm{G}-\mathrm{N})$ equation is not irrotational, and there lacks a systematic procedure for higher-order improvements. (However, Shields 1986 has proposed a varied approach to 'direct theory' including the higher orders.) Some simple forms of the $\mathrm{G}-\mathrm{N}$ model have been applied by Ertekin (1984) and by Ertekin, Webster \& Wehausen $(1984,1986)$ to problems of soliton generation, with results compared to other theoretical models.

In addition to these models, the forced Korteweg-de Vries (fKdV) equation was applied by Akylas (1984) and Cole (1985) to the case of a pointed surface-pressure forcing. Grimshaw \& Smyth (1986), Smyth (1987), Zhu (1986), Zhu, Wu \& Yates (1986, 1987), and Melville \& Helfrich (1987) derived the forced KdV equation for various cases and applied it to evaluate the internal solitary waves generated in a density-stratified layer of water by moving disturbances. These papers are of course quite general and not restricted only to studies of internal solitary waves.

The problem of generation of nonlinear waves in two horizontal space dimensions has been investigated by Mei (1986) based on approximations for slender bodies in a shallow channel, by Ertekin et al. (1986) employing Green-Naghdi's theory for three-dimensional calculations, by $\mathrm{Wu} \& \mathrm{Wu}$ (1987) based on the generalized Boussinesq model, and has also been evaluated by Katsis \& Akylas (1987) using the Kadomtsev-Petviashvili (K-P) model. These studies showed that a slender ship, or a surface-pressure patch of rectangular planform moving near the critical speed in a shallow channel of finite width can periodically radiate upstream-advancing solitons with crests stretched straight across the channel. In several specific cases, comparisons between the numerical results carried out using the various theoretical models indicate that the main features of the solutions appear to be in good agreement. Although a few attempts were also made in these studies to compare theory and experiment, only qualitative agreement between them can be inferred because no cases have so far been available with exactly the same basis to match the comparison.

The purpose of the present work is to carry out a joint numerical and experimental investigation of the generation of nonlinear dispersive waves by two-dimensional disturbances in the form of a submerged topography or a surface-pressure patch moving with a transcritical speed in a shallow channel. Both the g-B model and the fKdV model are employed for numerical studies, which include calculations of several derived flow quantities. A finite-difference numerical method similar to the original scheme introduced by $W u \& W u(1982)$ is adopted for computation over a range of the depth Froude number. The phenomenon of precursor solitions is predicted by both models; the results from the two models are qualitatively similar within a certain range of transcritical speeds, in which the generation process is shown to continue periodically and indefinitely. Some more refined differences between the two models will be examined later.

Experiments were conducted for a two-dimensional topography moving with a uniform transcritical velocity along the floor of a water tank. Comparison between experimental and numerical results of the two models investigated shows that the agreement is generally very good, in terms of both the amplitude and the phase of the waves generated, especially at critical and near critical speeds. As the Froude 


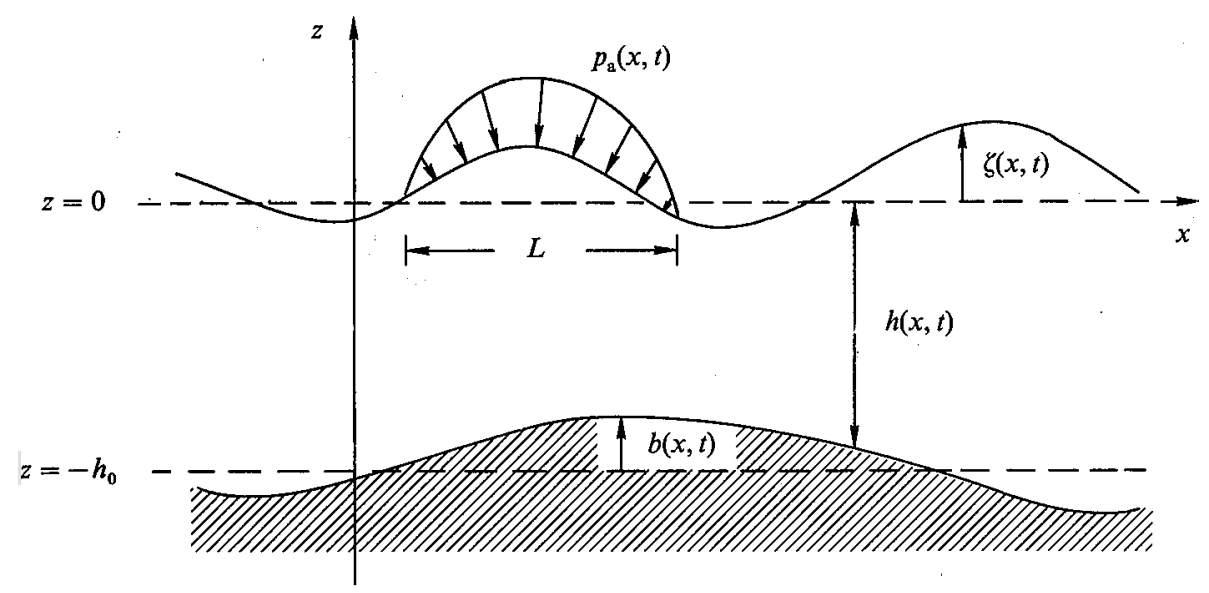

Frgure 1. The coordinate system and the two types of forcing agencies under consideration: the surface pressure $p_{\mathrm{a}}(x, t)$ and bottom topography $b(x, t)$.

number departs from 1, the fKdV model is found to increasingly overpredict, but only slightly the experimental results, while the g-B model consistently underestimates, very slightly, the wave amplitude for the case of a submerged topography. Wave breaking was observed experimentally in the leading precursor solitary waves at low supercritical speeds and in the leading trailing waves at high subcritical speeds for sufficiently strong disturbances. The mechanism responsible for the breaking of these waves lies beyond what the theoretical models are intended to predict and is left for future studies.

\section{The theoretical models}

For investigating the problem of generation of nonlinear waves on shallow water by forcing disturbances moving through the water layer, we shall adopt both the model proposed by $\mathrm{Wu}(1979,1981)$ and the $\mathrm{KdV}$ model used by Lee $(1985)$ and $\mathrm{Wu}$ (1987). Both models admit forcing functions in the form of a surface pressure and a submerged topography (see figure 1). The fluid, initially at rest under gravity and occupying a layer of uniform depth $h_{0}$, is assumed inviscid and incompressible and the resulting motion, irrotational. Two-dimensional motions of this kind are governed by the Laplace equation for the velocity potential $\phi(x, z, t)$, and the boundary conditions at the free surface and the bottom are given by

$$
\begin{aligned}
\epsilon \phi_{x x}+\phi_{z z} & =0, \quad-1+b(x, t)<z<\zeta(x, t), \\
\phi_{z} & =\epsilon\left(\zeta_{t}+\phi_{x} \zeta_{x}\right) \quad \text { on } \quad z=\zeta(x, t), \\
\zeta+\phi_{t}+\frac{1}{2}\left(\phi_{x}^{2}+1 / \epsilon \phi_{z}^{2}\right) & =-p_{\mathrm{a}}(x, t), \quad z=\zeta(x, t), \\
\phi_{z} & =\epsilon\left(b_{t}+\phi_{x} b_{x}\right), \quad z=-1+b(x, t),
\end{aligned}
$$

where the horizontal coordinate $x$ is scaled by a typical wavelength $\lambda$, the vertical coordinate $z$ by the constant undisturbed water depth $h_{0}$, and the time $t$ by $\lambda / c_{0}$; $c_{0}=\left(g h_{0}\right)^{\frac{1}{2}}$ is the characteristic critical speed, and $g$ is the gravitational acceleration. The free surface elevation $\zeta$ and the topography $b(x, t)$ are scaled by $h_{0}$, the velocity potential $\phi(x, z, t)$ by $c_{0} \lambda$, and the pressure $p(x, z, t)$ and surface pressure $p_{\mathrm{a}}(x, t)$ by 
$\rho g h_{0}$. From the normalization, two important parameters arise, with $\epsilon=\left(h_{0} / \lambda\right)^{2}$ providing a measure of the dispersive effects and with $\alpha=a / h_{0}$, where $a$ is a typical wave amplitude, giving an estimate of the nonlinear effects. For long-wave theory, $\epsilon \ll 1$; and the nonlinear and dispersive effects are brought into a proper balance, such as for the Boussinesq class and the present work, if

$$
\epsilon=\left(\frac{h_{0}}{\lambda}\right)^{2} \ll 1, \quad \alpha=\frac{\alpha}{h_{0}} \ll 1, \quad \alpha=O(\epsilon) .
$$

The above scaling implies that in the wave frame of reference following a primary wave the normalized quantities $\zeta, \phi, \phi_{t}$, and $\phi_{x}$ are all $O(\alpha)$. The two external forcing disturbances, namely the surface pressure $p_{\mathrm{a}}(x, t)$ and topography $b(x, t)$, are further assumed to be small and slowly varying functions such that

$$
\left|p_{\mathrm{a}}\right|,|b|=O(\alpha) ; \quad\left|b_{x}\right|,\left|b_{t}\right|,\left|p_{\mathrm{a} x}\right|,\left|p_{\mathrm{a} t}\right| \leqslant O(\alpha) .
$$

By using the expansions of $\phi(x, z, t)$ and $\zeta(x, t)$ in a power series of $\epsilon$, the generalized Boussinesq equations are derived as follows (see Wu 1979, 1981; Lee 1985),

$$
\begin{aligned}
& \zeta_{t}+\left[(1-b+\zeta) \bar{\phi}_{x}\right]_{x}=b_{t}, \\
& \zeta+\bar{\phi}_{t}+\frac{1}{2} \bar{\phi}_{x}^{2}-\frac{1}{3} \epsilon \bar{\phi}_{x x t}=-p_{\mathrm{a}}-\frac{1}{2} e b_{t t},
\end{aligned}
$$

where $\bar{\phi}$ is the layer-mean velocity potential defined as

$$
\bar{\phi}(x, t)=\frac{1}{1-b+\zeta} \int_{-1+b}^{\zeta} \phi(x, z, t) \mathrm{d} z .
$$

Equations (7) and (8) have a relative error (with respect to the leading term) of $O\left(\alpha \epsilon, \epsilon^{2}\right)$. The original set of equations obtained by Wu $(1979,1981$, equations (41), (42) therein), based on the less restrictive assumption that $b_{x}=O(1)$, reduce to (7) and (8) if, in $h=h_{0}-b, b$ is taken of $O(\alpha)$ and its dependence on the third dimension $y$ is removed.

As a variant of the above two-equation $\mathrm{g}-\mathrm{B}$ model, (7) and (8) can be readily combined by eliminating $\zeta(x, t)$ to yield a single fourth-order partial differential equation for $\bar{\phi}$ as

$$
\bar{\phi}_{t t}-\bar{\phi}_{x x}+2 \bar{\phi}_{x} \bar{\phi}_{x t}+\bar{\phi}_{t} \bar{\phi}_{x x}-\frac{1}{3} \epsilon \bar{\phi}_{x x t t}=-\left(p_{\mathrm{a}}+b\right)_{t}-\left[\left(p_{\mathrm{a}}+b\right) \bar{\phi}_{x}\right]_{x}-\frac{1}{2} \epsilon b_{t t t}
$$

In this generalized Boussinesq single-equation model, the two disturbances appear to their leading order in simple additive form $\left(p_{\mathrm{a}}+b\right)$, relative to which the last term in (10), or the $b_{t t}$ term in (8) is higher in order and is further indicative that, of the two disturbances, the moving topography bears additional dispersive effects. Equation (10) suggests that a surface pressure would produce a quite similar solution in comparison to a bottom topography of the same distribution; however, (10) is deduced from (7) and (8) with further approximation of the Boussinesq class and is numerically not identical with the system (7) and (8). Lepelletier (1981) and Schember (1982) derived a similar equation under the same assumptions as $\mathrm{Wu}$ (1979), which was used in their numerical studies.

The KdV class of equation with imposed forcing can be derived from the above g$B$ model by restricting the wave propagation to be unidirectional. There are several different methods of derivation, one using a particular slow timescale for describng the motion as viewed in the wave frame, as shown by Grimshaw \& Smyth (1986) and $\mathrm{Wu}$ (1987), and another being based on consistency of the order expansion as 
illustrated by Whitham (1974) for the homogeneous case. We present below a derivation along the second approach, which has some interesting features of its own.

First we differentiate (8) with respect to $x$ and modify the result and (7) as

$$
\begin{aligned}
\zeta_{t}+[(1-\alpha b+\alpha \zeta) \bar{u}]_{x} & =\alpha b_{t}, \\
\zeta_{x}+\bar{u}_{t}+\alpha \bar{u} \bar{u}_{x}-\frac{1}{3} \epsilon \bar{u}_{x x t} & =-\alpha p_{\mathrm{a} x}
\end{aligned}
$$

with $\bar{u}$, the layer-mean horizontal velocity component, replacing $\partial \bar{\phi} / \partial x$, the $x$ derivative of the layer-mean velocity potential, which restores the original exact continuity equation (11) (Wu 1981, equation (37)). Further, we have inserted in (11) and (12) factors of $\alpha$ to indicate, for convenience of operation, the order of the respective terms with reference to the leading terms. So that no secular terms will arise in the following expansion, the forcing functions are taken $O\left(\alpha^{2}\right)$.

First, the arbitrary $p_{\mathrm{a}}$ and $b$ are required to move near the critical speed, and hence for left-moving disturbances we take

$$
p_{\mathrm{a}}=p_{\mathrm{a}}(x+(1+\delta) t), \quad b=b(x+(1+\delta) t),
$$

where $\delta$, the detuning parameter, is $O(\alpha)$ and $b_{t}$ can be replaced by $b_{x}$ with a relative error of $O(\alpha)$. Now, to the lowest order, (11) and (12) reduce to

$$
\zeta_{t}+\bar{u}_{x}=0, \quad \zeta_{x}+\bar{u}_{t}=0,
$$

which gives

$$
\bar{u}=-\zeta, \quad \zeta_{t}-\zeta_{x}=0
$$

Seeking a solution, correct to the two leading orders in $\alpha$, in the form

$$
\bar{u}=-\zeta+\alpha A+O\left(\alpha^{2}\right)
$$

with $A$ assumed a function of $\zeta$ and its derivatives, (11) and (12) become

$$
\begin{aligned}
\zeta_{t}-\zeta_{x}+\alpha\left(A_{x}-2 \zeta \zeta_{x}-b_{t}\right) & =O\left(\alpha^{2}\right), \\
\zeta_{t}-\zeta_{x}-\alpha\left(A_{t}+\zeta \zeta_{x}+\frac{1}{3} \hat{e} \zeta_{x x t}+p_{\mathrm{a} x}\right) & =O\left(\alpha^{2}\right),
\end{aligned}
$$

where $\hat{e}=\epsilon / \alpha=O(1)$. By using (15) in the second-order terms, (17) and (18) are consistent if

$$
A=\frac{1}{4} \zeta^{2}-\frac{1}{6} \hat{e} \zeta_{x t}+\frac{1}{2}\left(b-p_{\mathfrak{a}}\right),
$$

by which (17) and (18) coincide and they together with (16) give

$$
\begin{gathered}
\zeta_{t}-\left(1+\frac{3}{2} \alpha \zeta\right) \zeta_{x}-\frac{1}{6} \epsilon \zeta_{x x x}=\frac{1}{2} \alpha\left(p_{\mathrm{a}}+b\right)_{x}, \\
\bar{u}=-\zeta+\frac{1}{4} \alpha \zeta^{2}-\frac{1}{6} \epsilon \zeta_{x x}+\frac{1}{2} \alpha\left(b-p_{\mathrm{a}}\right) .
\end{gathered}
$$

Equation (20) is the fKdV equation sought. Equation (21), similar to a Riemann invariant, provides the layer-mean velocity to the same order as $\zeta$ given by (20). These results, with an error term of $O\left(\alpha^{2}\right)$, agree with $\mathrm{Wu}(1987)$ for arbitrary $p_{\mathrm{a}}$ and $b$ distributions. Note that for right-going waves we only need to change the sign of the $\zeta_{t}$ term in (20) and the sign of $\bar{u}$ in (21). Finally, the problem is completed by specifying initial conditions for $\zeta$ and $\bar{u}$ at $t=0$. We may further set $\alpha$ and $\epsilon$ to unity by rescaling. 


\section{Wave resistance and work-energy relation}

A disturbance moving through an inviscid fluid having a free surface generally experiences a resistance due to generation and radiation of surface waves. This resistance is the streamwise component of the resultant hydrodynamic pressure acting on the moving body surface, which in coefficient form, for the present twodimensional case for a left-going disturbance, is

$$
C_{D_{\mathrm{W}}}=\frac{D_{\mathrm{w}}}{\rho g h_{0}^{2}}=\int_{-\infty}^{\infty}\left(-p_{\mathrm{a}} \zeta_{x}+p_{\mathrm{b}} b_{x}\right) \mathrm{d} x
$$

where $p_{\mathrm{b}}=p\left(x,-h_{0}+b, t\right)$ denotes the hydrodynamic pressure acting on the topography surface and we have rescaled so that $\alpha=\epsilon=1$.

To maintain a specified motion of the disturbance, a mechanical power is required. By the energy principle for the present case of an incompressible and inviscid fluid, the mechanical power input is equal to the rate of increase in the total energy of the system, $E$, so that

$$
\frac{\mathrm{d} E}{\mathrm{~d} t}=-\int_{V} \boldsymbol{u} \cdot(\boldsymbol{\nabla} p) \mathrm{d} V=-\int_{V} \boldsymbol{\nabla} \cdot(p \boldsymbol{u}) \mathrm{d} \boldsymbol{V},
$$

where $E$ is the integral of $\left[\frac{1}{2}(\nabla \phi)^{2}+z\right]$ throughout the fluid volume $V$. Accordingly, for the configuration at hand,

$$
\begin{aligned}
\frac{\mathrm{d} E}{\mathrm{~d} t} & =-\int_{-\infty}^{\infty} \mathrm{d} x \int_{-h}^{\zeta}\left[(p u)_{x}+(p w)_{z}\right] \mathrm{d} z, \\
& =-\int_{-\infty}^{\infty}\left[\int_{-h}^{\zeta}(p u) \mathrm{d} z\right]_{x} \mathrm{~d} x+\int_{-\infty}^{\infty}\left\{\left[p\left(u \zeta_{x}-w\right)\right]_{z=\zeta}-\left[p\left(u b_{x}-w\right)\right]_{z=-h}\right\} \mathrm{d} x .
\end{aligned}
$$

The first integral in the last expression vanishes because of the boundary conditions at infinity, so that by making use of the boundary conditions (2) and (4) we have

$$
\frac{\mathrm{d} E}{\mathrm{~d} t}=-\int_{-\infty}^{\infty}\left(p_{\mathrm{a}} \zeta_{t}-p_{\mathrm{b}} b_{t}\right) \mathrm{d} x
$$

In the body frame of reference $\left(x^{\prime}, z^{\prime}, t^{\prime}\right)$ moving uniformly to the left with the Froude number $F=U / c_{0}$ of the disturbance, where

$$
x^{\prime}=x+F t, \quad z^{\prime}=z, \quad t^{\prime}=t,
$$

we have $\zeta_{t}=\zeta_{t^{\prime}}+F \zeta_{x^{\prime}}$, and a similar expression for $b_{t}$. Hence the work-energy relation (25) becomes

where

$$
\begin{aligned}
\dot{E} & =F C_{D_{\mathrm{w}}}+\dot{E}_{\mathrm{v}}, \\
\dot{E}_{\mathrm{v}} & =\int_{B}\left(-p_{\mathrm{a}} \zeta_{t^{\prime}}+p_{\mathrm{b}} b_{t^{\prime}}\right) \mathrm{d} x^{\prime},
\end{aligned}
$$

represents the rate of work due to any vertical wave motion underneath the surface pressure and any vertical movement of the submerged topography apparent to the moving frame. Note that the contribution to $\dot{E}_{\mathrm{v}}$ from the term with $p_{\mathrm{b}}$ vanishes if the topography is restrained from vertical movement in the body frame.

Finally, the pressure $p_{\mathrm{b}}$ is obtained from the Bernoulli equation applied to the upper surface of the topography,

$$
p_{\mathrm{b}}=1-b-\phi_{t}+O\left(\epsilon^{2}\right) .
$$


This equation, when combined with the Bernoulli equation applied to the free surface,

$$
\phi_{t}=-\zeta-p_{\mathrm{a}}+O\left(\epsilon^{2}\right),
$$

and noting that to leading order $\phi$ is independent of $z$, gives the pressure on the bottom as

$$
p_{\mathrm{b}}=1+\zeta-b+p_{\mathrm{a}}+O\left(\epsilon^{2}\right),
$$

which is the hydrostatic pressure to the order retained. For the fKdV model, $p_{\mathrm{a}}$ and $b$ are to be neglected in (31) for consistency.

\section{Numerical results and discussion}

Although the two models developed in $\$ 2$ belong to the same class as the original Boussinesq model, all being characterized by (5), it is of interest to compare their numerical results, namely, the results of the g-B equations (7) and (8) and the fKdV equation (20). In both cases, finite-difference methods based upon the improved numerical scheme employed by $\mathrm{Wu} \& \mathrm{Wu}(1982)$ are adopted.

For reasons of numerical stability in solving the fKdV equation, as first pointed out by Benjamin, Bona \& Mahony (1972), it is preferred to replace the dispersion term $\zeta_{x x x}$ with $\zeta_{x x t}$, which is obtained by using the lowest-order approximation (see (15)) for this modification. For both the fKdV and g-B equations, forward differences are used for time derivatives and central differences for spatial derivatives. The resulting difference equations are solved in a finite spatial domain $\left(x_{m} \leqslant x \leqslant x_{M}\right)$, which is called a window; and the uniform grid sizes in space and in time are denoted by $\Delta x$ and $\Delta t$, respectively. A predictor-corrector method is employed without iteration at the corrector stage, and both stages reduce to the inversion of a constant tridiagonal matrix, which is strictly diagonally dominant (for details see Lee 1985).

To keep the computational window reasonably small, we introduce a pseudomoving frame, which moves in the same direction as the disturbance. The reference frame is fixed with respect to the fluid (at rest) at $x= \pm \infty$, and only the region of computation undergoes stepwise shifts; hence the name pseudo-moving frame. The boundary conditions imposed on the computational boundaries are called open or transparent boundary conditions, and they are intended to permit the passage of a wave through the boundaries with as little non-physical reflections from the boundaries as possible in order not to contaminate the region of computation beyond a certain error limit. We use

$$
\zeta_{t}= \pm \zeta_{x}
$$

where the sign is taken for each window border so that the waves always propagate out of the window.

If $\Delta t$ and $\Delta x$ are chosen such that

$$
J=\frac{\Delta x}{F \Delta t}
$$

is an integer greater than $1(J=2,3,4, \ldots)$, then the disturbance travels a distance $\Delta x$ in $J$ timesteps. If the window is shifted every $J$ timesteps and if we view the solution only when the window is just shifted, the position of the disturbance in the window will not change. At each time of window shifting, a new grid point emerges into view from the upstream side of the window while an old grid point disappears at the downstream side as it moves behind the window. In this way the mechanical laws are always referred to the fluid frame, and only the region of computation 
undergoes stepwise changes. As the shifting is done in the direction of the motion of the disturbance, the downstream boundary always chases any non-physical waves reflected from this boundary due to any imperfectness of the open boundary condition. If we assume that the reflected waves are small in magnitude but remain long in wavelength, they must propagate with a speed close to the critical speed. Therefore, when disturbances move with $F \approx 1.0$, the reflected waves from the downstream boundary cannot move far from the boundary with the window shifting. The upstream boundary is taken far enough away from the forerunning waves so that no waves of significant size encounter that boundary. To provide the new upstream boundary value of $\zeta$ when the window is shifted, we use the same principle used for the open boundary condition (32).

The stability and the convergence of the code was first tested for the homogeneous case, $p_{\mathrm{a}}=b=0$, with a prescribed initial wave which is a solution of permanent form. In this way we were able to ascertain the best range of $\Delta x$ and $\Delta t$, and good results were obtained when compared to exact solutions for the KdV equation and to other numerical results for the Boussinesq equations. For the case of inhomogeneous problems, $J$ in (33) is chosen for given $F$ as an integer so that

$$
J F=\frac{\Delta x}{\Delta t} \approx 2 .
$$

Although our numerical scheme is implicit, for convergence it is generally believed safe to maintain the Courant-Friedrichs-Lewy condition and to allow a margin to account for possible departure of the condition due to the effects of nonlinearity. We obtained satisfactory results for $\Delta x=0.2$ and $\Delta t$ determined by (34) and used these values for most of the results presented in this study.

We first consider a surface pressure distribution of the form

$$
p_{\mathrm{a}}(X, T)=p_{\mathrm{m}} \sin ^{2}\left[\frac{\pi}{L}(X+F T)\right] \text { for } \quad 0 \leqslant(X+F T) \leqslant L,
$$

and $p_{\mathrm{a}}=0$ elsewhere, where $L$ is the length of the disturbance referenced to $h_{0}, X$ is referenced to $h_{0}$ and $T$ to $h_{0} / c_{0}$. The motion starts impulsively from rest at $T=0$, and $X=0$ is taken at the initial leading edge of the disturbance. The water surface elevation and the wave resistance coefficient were computed for various values of $F(0.1-1.3)$ and $p_{\mathrm{m}}(=0.05,0.1,0.15)$. While the lower range of $F=1+\delta$ down to 0.1 is admittedly quite beyond the appropriate detuning range originally intended for $\delta$ as indicated in (13) for the fKdV model, this range is nevertheless included in our calculations for the sake of making comparisons between $(a)$ the fKdV model, $(b)$ the g-B model, for which $F$ is not restricted to be near 1 , and $(c)$ the experimental data. Results of the fKdV model for $p_{\mathrm{m}}=0.1$ are shown in figure 2 for three different Froude numbers. In each case the upper plot shows the development of the surface wave elevation with time as viewed in the body frame and the lower curve gives the wave resistance coefficient $C_{D_{\mathrm{W}}}$ as a function of time.

For low subcritical $(F<0.2)$ and high supercritical speeds $(F>1.2)$, the initial transient period quickly passes, and only local waves near the disturbance remain to appear stationary with respect to the moving disturbance. In these cases, the surface elevations predicted by the inhomogeneous linear non-dispersive long-wave equation are found to be satisfactory for sufficiently weak disturbances (with only a slight underprediction of the wave elevation, see $W u \& W u$ 1982). Within the Froude number range $0.2-1.2$, the wave pattern is drastically different from that predicted 

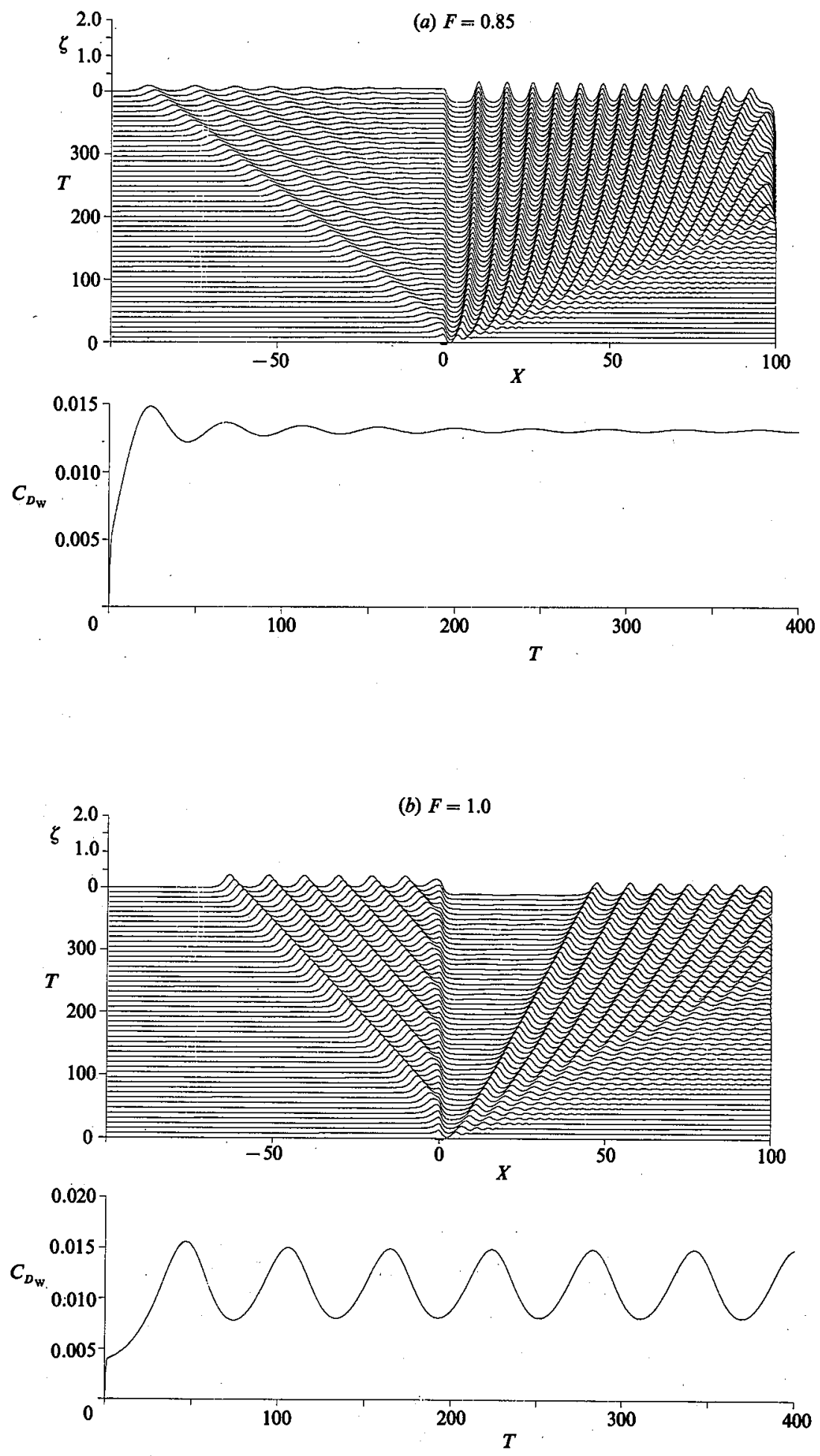

Figure $2(a, b)$. For caption see facing page. 

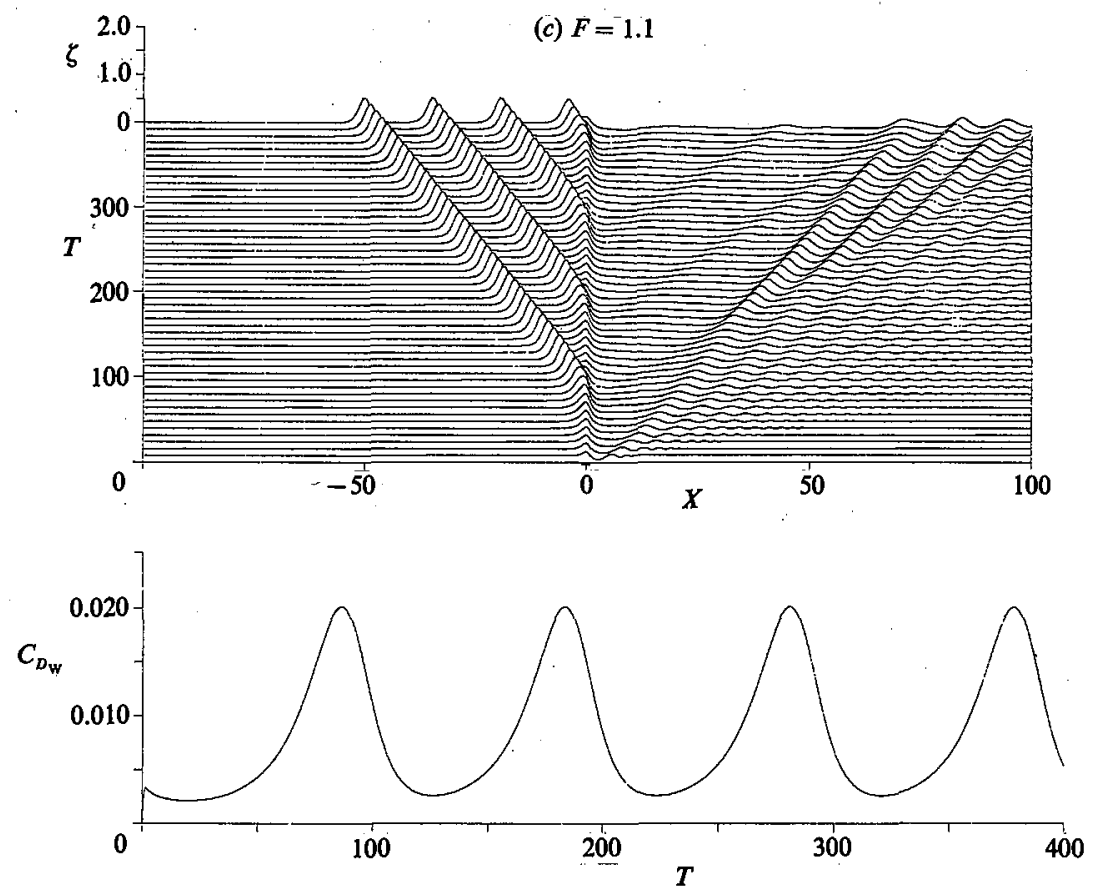

FIgURE 2. The upper plot shows numerical results for the surface elevation $\zeta$ (based on the fKdV model) as a function of $X$ and $T$ for the surface pressure distribution (35), with $p_{\mathrm{m}}=0.1$ and $L / h_{0}=2.0 ;(a) F=0.85,(b) F=1.0,(c) F=1.1$. The wave heights are given in the disturbance frame and the position of the disturbance is indicated by a solid bar on the $T=0$ profile. The lower plot gives the wave resistance coefficient as a function of time.

by the linear dispersive theory. Positive waves are observed running ahead of the disturbance, a phenomenon that is not possible in classical linear theory. For the transcritical range of Froude number $(F \approx 1)$, the present nonlinear theory predicts, unlike the well-known singular state given by the linear dispersive theory (cf. Lamb $1932, \S 177$ ), well-defined waves both leading and trailing the moving disturbance, which can be delineated as follows.

For subcritical speeds $(0.25 \leqslant F \leqslant 0.85)$, the trailing waves near the disturbance change only very slowly, but there is a series of positive waves moving ahead of the disturbance, which are small in magnitude compared with the trailing waves. At transcritical speeds $(0.85<F<1.15)$, a series of large positive waves run ahead of the disturbance and a slowly prolonging negative wave of nearly constant depressed surface develops immediately behind the disturbance. This depressed region, which is pronounced in length only at near transcritical speeds, is then followed by a train of modulated cnoidal waves. Both the amplitude of the upstream-advancing waves $a_{\mathrm{s}}$ and their generation period $T_{\mathrm{s}}$ are increasing functions of $F$ (figure 3 ). At low Froude numbers precursor waves are produced more rapidly; however, they are of relatively small amplitude. Above $F \approx 0.8, a_{\mathrm{s}}$ and $T_{\mathrm{s}}$ increase very rapidly as $F$ approaches the limiting value of about 1.2 in this case. Figures 3-6 were compiled from numerical results of the $\mathrm{g}-\mathrm{B}$ equations with a pressure distribution given by (35).

For making comparison between the forerunning and trailing waves, we define $r_{a}$ as

$$
r_{a}=\frac{a_{\mathrm{s}}}{a_{\mathrm{t}}}
$$




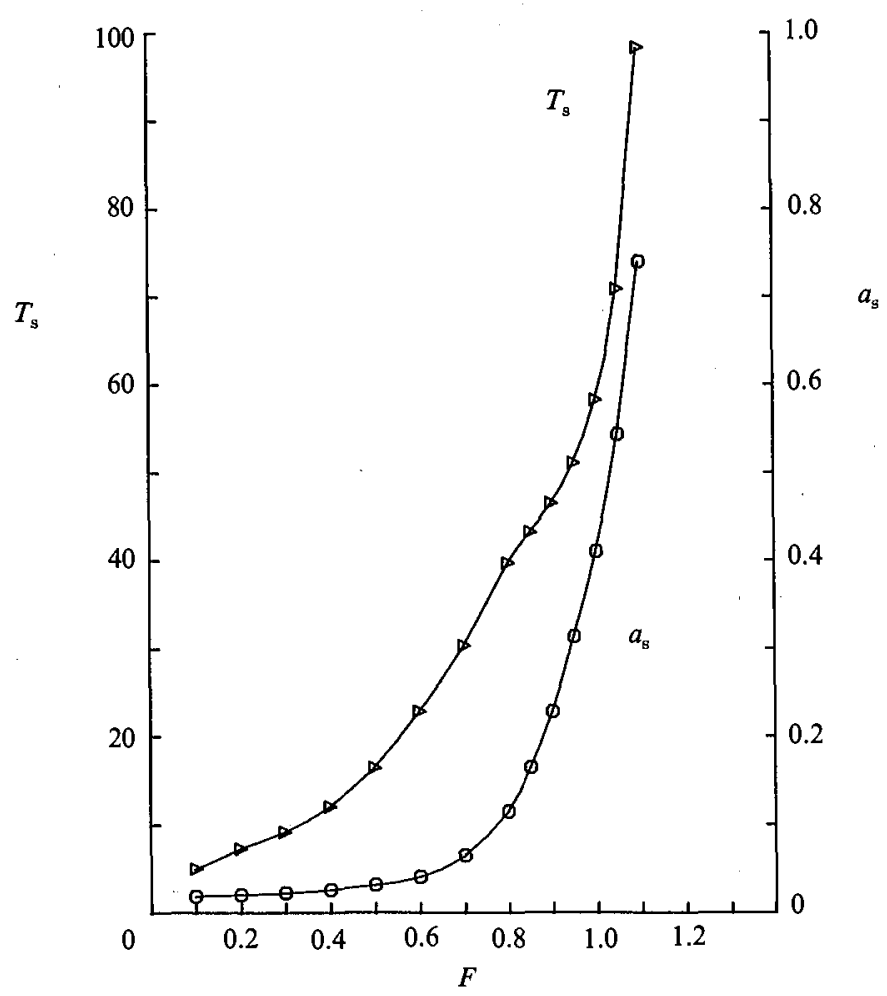

FIGURE 3. The amplitude $a_{\mathrm{s}}$ and the generation period $T_{\mathrm{s}}$ of the first precursor solitary wave obtained, numerically from the $\mathrm{g}-\mathrm{B}$ equations, are shown for the pressure distribution (35) as a function of Froude number $F ; p_{\mathrm{m}}=0.1$ and $L / h_{0}=2.0$

where $a_{\mathrm{t}}$ is the wave height of the undulating trailing wave closest to the disturbance. As $F$ is increased, $r_{a}$ grows as long as the forward-running waves are generated (see figure 4). This trend of increasing $r_{a}$ illustrates the differences between sub- and transcritical speeds; it summarizes the outstanding feature that for small $F$ the trailing waves are the most striking, whereas at higher $F$ the leading waves grow to substantial size while the trailing waves subside.

For other values of the $p_{m}(0.05-0.2)$, the general trends discussed above are all similar and the dependence of $T_{\mathrm{s}}$ on $p_{\mathrm{m}}$ is presented in figure 5 . Here we see that, as $p_{\mathrm{m}}$ increases, $T_{\mathrm{s}}$ decreases and the upper bound of $F$, above which no solitons are generated, becomes slightly larger.

The overall features of the wave elevations outlined above are in agreement with similar numerical results obtained by others (Akylas 1984; Cole 1985; Mei 1986; Grimshaw \& Smyth 1986; Melville \& Helfrich 1987) for slightly different flow parameters and forcings, some using the singular delta function as the forcing. Relatively few previous works have examined the wave resistance (Lee 1985; Ertekin et al. 1986; Wu 1987), and from our numerical results corresponding to the cases described above, we have seen that for $0.2<F<1.2, C_{D_{\mathrm{W}}}(t)$ has as many maxima as the number of upstream-advancing waves. From the wave resistance plots (lower curves in figure 2) we observe that each relative maximum of $C_{D_{\mathrm{w}}}$ corresponds to the 'birth' of a new precursor wave. Thus, the generation period of these waves is easily identified by the oscillations of the wave resistance. For $F<0.8$, we observe that the oscillatory part of $C_{D_{\mathrm{w}}}$ is very small compared with its time- 


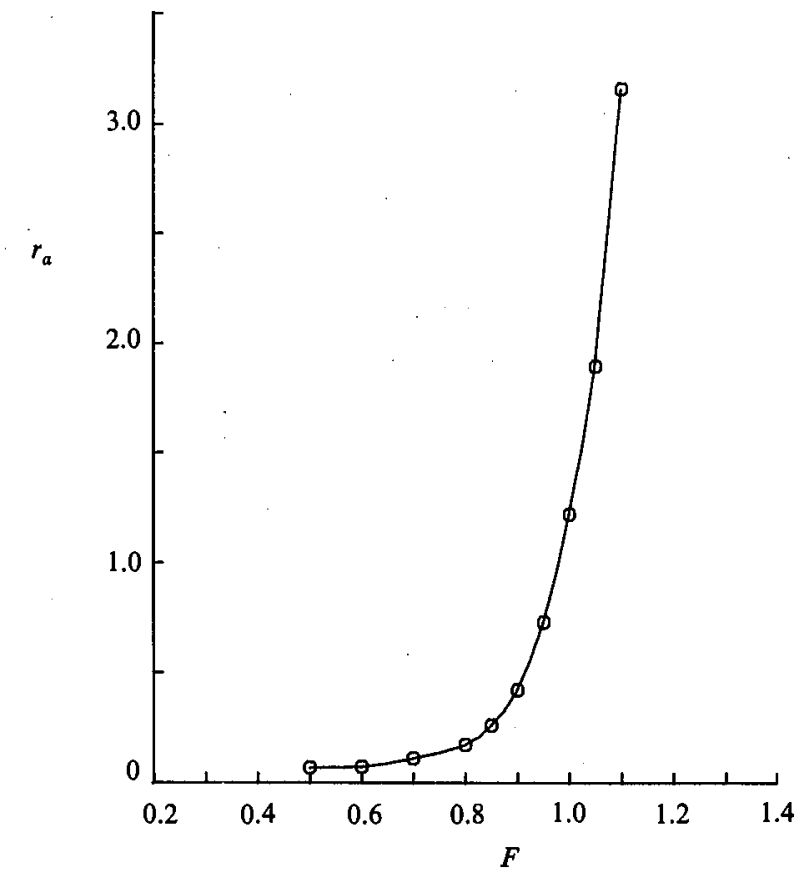

FIGURE 4. The ratio of the amplitude of the first precursor solitary wave and the wave height of the first trailing wave crest is shown as a function of the Froude number, based on the g-B equations for the pressure distribution (35) with $p_{\mathrm{m}}=0.1$ and $L / h_{0}=2.0$.

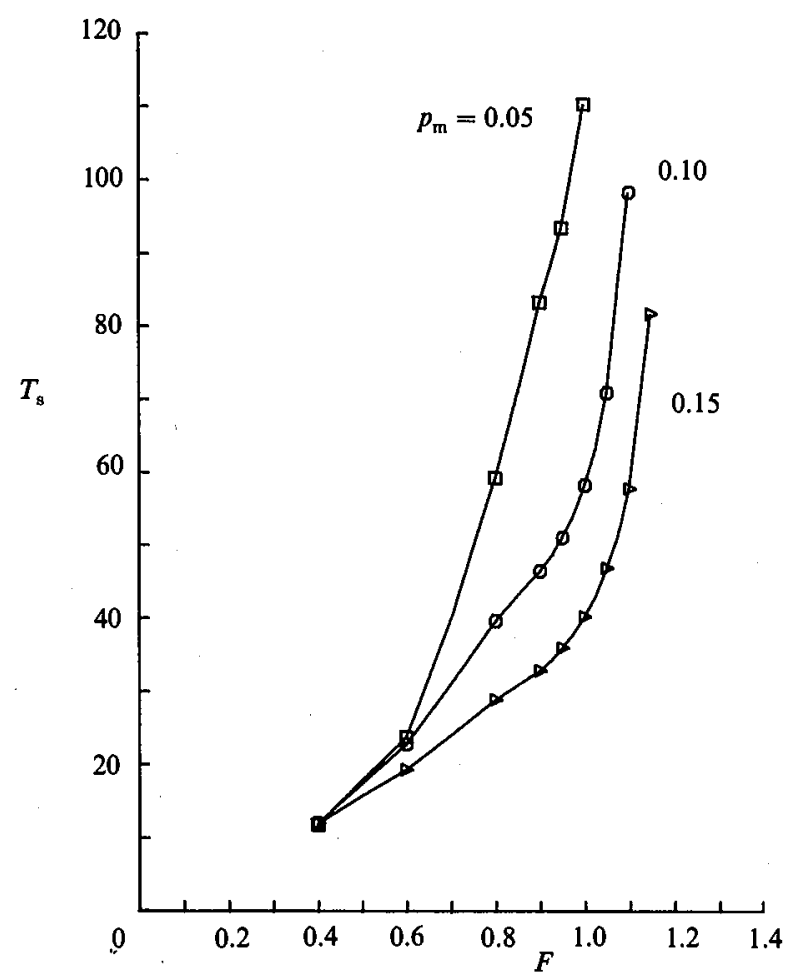

Figure 5. The generation period $T_{\mathrm{s}}$ of the first precursor wave is plotted versus Froude number for $p_{\mathrm{m}}=0.05,0.1$ and 0.15 , based on the $\mathrm{g}-\mathrm{B}$ equations for the pressure distribution (35) with $L / h_{0}=2.0$. 


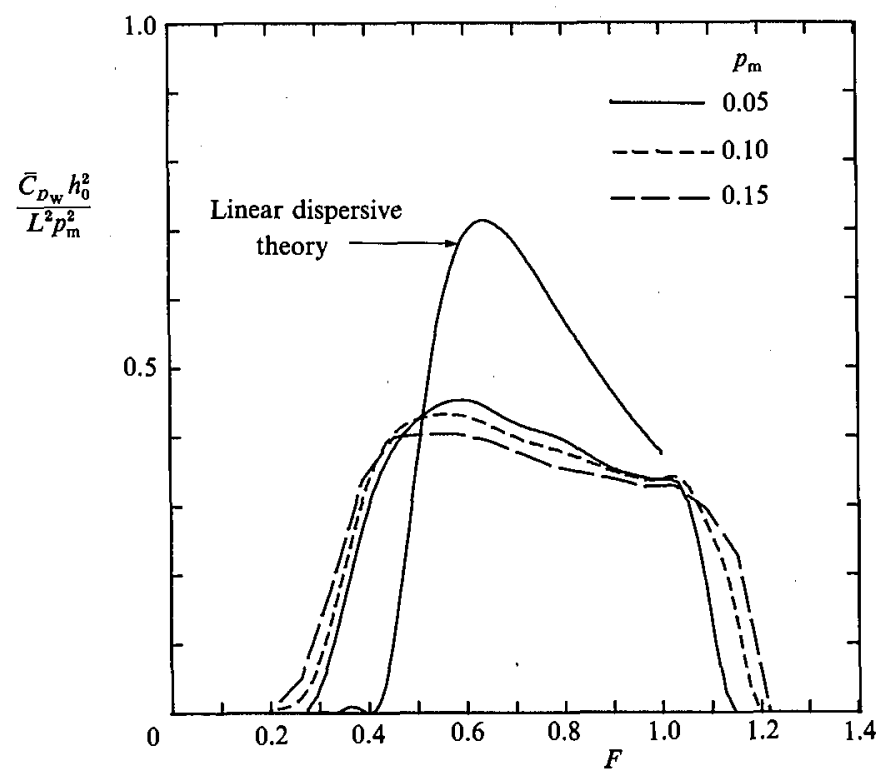

FIGURE 6. The normalized wave resistance coefficients for the linear dispersive and nonlinear models are plotted as functions of the Froude number; all cases are for the pressure distribution (35) with $L / h_{0}=2.0$. The linear dispersive theory is from (37), and the nonlinear data are based on the $\mathrm{g}$-B equations with $p_{\mathrm{m}}=0.05,0.10$, and 0.15 .

average value $\bar{C}_{D_{\mathrm{w}}}$. For the subcritical case $(F<1)$, the difference between a maximum and the subsequent minimum values of $C_{D_{\mathrm{w}}}$ decreases with time. This may imply that the generation of the runaway solitons might cease to be discernible after a certain period of time or it may settle to a reduced level. We also note that the upstream surface level near the leading edge of the disturbance rises, very gradually, to a certain value. The time required to reach such a limiting state becomes longer as $F$ approaches 1, while the runaway solitons become larger, and the depressed region just behind the disturbance becomes more pronounced. For $F$ very close to 1 , the fluctuations in $C_{D_{\mathrm{w}}}$ are nearly as large as $\bar{C}_{D_{\mathrm{w}}}$ and the wave amplitude of the runaway solitons is about the same as the wave height of the trailing waves. Furthermore, the difference between a maximum and the following minimum changes very little as time increases, thus implying that the generation of the runaway solitons may continue indefinitely. For $F$ greater than 1.2 approximately, the precursor solitons are unable to propagate away from the disturbance, and the wave evolution ceases, so that waves appear only localized in the neighbourhood of the disturbance.

The average wave resistance coefficient $\bar{C}_{D_{\mathrm{w}}}$ was evaluated from the numerical results by averaging $C_{D_{\mathrm{W}}}$ over the time interval between the first and the third minimum (or fourth when available). The three $\bar{C}_{D_{\mathrm{W}}}$ curves, normalized by $p_{\mathrm{m}}^{2} L^{2} / h_{0}^{2}$, are plotted as a function of $F$ in figure 6 for $p_{\mathrm{m}}=0.05,0.1$, and 0.15 . They increase from zero at $F \approx 0.2$ to a maximum at $F \approx 0.5-0.6$, then decrease slightly as $F$ increases to 1.0 before falling off rapidly to zero in a narrow supercritical range. Also presented in figure 6 is the result of $\bar{C}_{D_{\mathrm{W}}}$ obtained from linear dispersive theory. Since linear dispersive theory for the steady-state limit predicts that the wave elevation becomes unbounded as $F$ approaches unity from below, it may seem surprising to see a maximum $\bar{C}_{D_{\mathrm{W}}}$ occurring near $F=0.6$ rather than 1 ; and to see the wave resistance 
reach a finite limit as $F$ tends to unity from below. In fact, the wave resistance for the pressure disturbance (35) can be found (see Lee 1985) as

$$
\bar{C}_{D_{\mathrm{W}}}=\frac{p_{\mathrm{m}}^{2} \sin ^{2}\left(\frac{k L}{2 h_{0}}\right)}{\left[1-\left(\frac{k L}{2 \pi h_{0}}\right)^{2}\right]^{2}\left[1-\left(\frac{2 k}{\sinh 2 k}\right)\right]},
$$

where the dimensionless wavenumber, $k=2 \pi h_{0} / \lambda$, is determined by the dispersion relation

$$
F^{2}=\frac{\tanh k}{k}
$$

There are two limiting cases of interest, one being when $F$ approaches zero from above and the other when $F$ approaches one from below; they are found from (37) to be zero and $\frac{3}{8}\left(p_{\mathrm{m}} L / h_{0}\right)^{2}$, respectively. Because $\bar{C}_{D_{\mathrm{w}}}$ is zero for $F>1$ in linear dispersive theory for the steady motion assumed, $\bar{C}_{D_{\mathrm{w}}}$ has a finite discontinuity at $F=1$.

The range of Froude numbers in which $\bar{C}_{D_{\mathrm{w}}}$ becomes appreciable in magnitude corresponds to the range over which precursor solitons are observed from both the nonlinear models and the experimental results. Although the linear theory gives fundamentally different wave patterns and fails to predict the upstream-advancing solitons altogether, the qualitative agreement in the prediction of the wave resistance coefficient, however, bears some crude resemblance as seen from figure 6 over the whole range of $F$. From figure 3 we see that for $F>0.6$ precursor solitons of noticeable size $\left(a_{\mathrm{s}} \geqslant 0.05\right)$ begin to form and clearly indicate the need for nonlinear models. As the strength of the disturbance is increased, the difference between the two theories becomes larger, as would be expected, and so does the range of $F$ over which the nonlinear theory predicts appreciable values of the wave resistance. Within the Froude-number range $0.2<F<0.6$, further experimental investigations will be required to provide an assessment of the different theories.

As can be seen from (10), the Boussinesq equations differentiate a moving surface pressure from a moving bottom topography of the same distribution, while the fKdV equation considers both as being equivalent. In figure 7 we compare the results of the g-B equations for a surface pressure (35), the g-B equations for a bottom topography with the same distribution, and the fKdV equation with the forcing (35). All the cases shown in figure 7 are computed at $F=1$ and with a disturbance strength of $p_{\mathrm{m}}=0.2$ or $b_{\mathrm{m}}=0.2$.

All three calculations reveal upstream-advancing solitary waves, a prolonging depressed region immediately behind the disturbance, and a train of trailing cnoidal waves. Refined differences are observed in the amplitude of the waves and the period of generation for upstream waves. For the $\mathrm{g}-\mathrm{B}$ model, the surface pressure acts as a relatively stronger disturbance than the bottom bump, thereby producing relatively larger waves in a shorter period. The $\mathrm{fKdV}$ model gives results intermediate between the two modes of forcing for the $\mathrm{g}-\mathrm{B}$ model. This basic feature holds for a wide range of values of $p_{\mathrm{m}}$ or $b_{\mathrm{m}}$ and Froude number.

\section{Mass-energy theorem}

As already noted and as illustrated in figure 8, there exist in the body frame of reference three uniform states of this transcritical phenomena of resonance: the first is the uniform free-stream state with water depth $h_{0}$ and velocity $U$ incident from the 
(a)

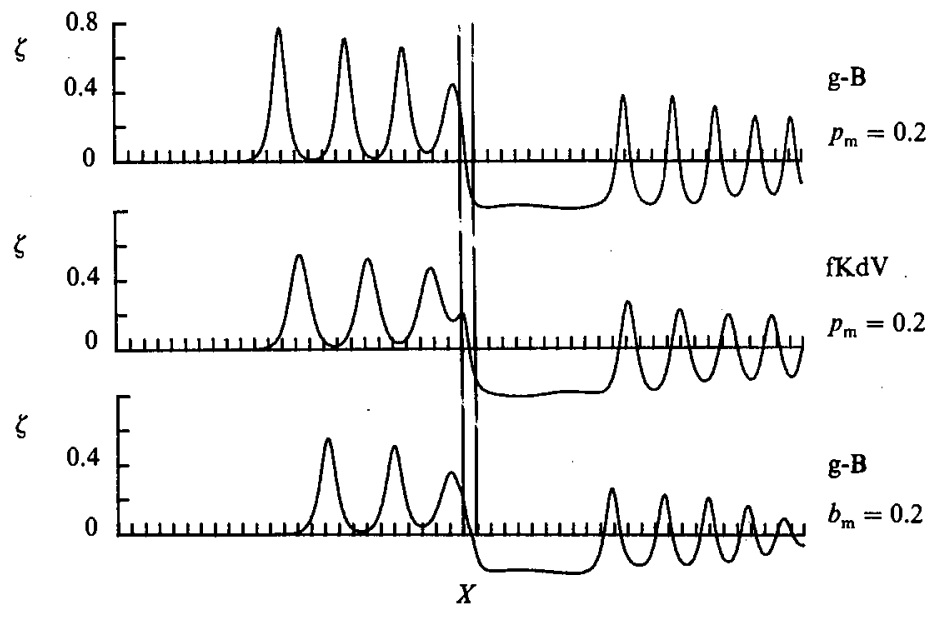

(b)
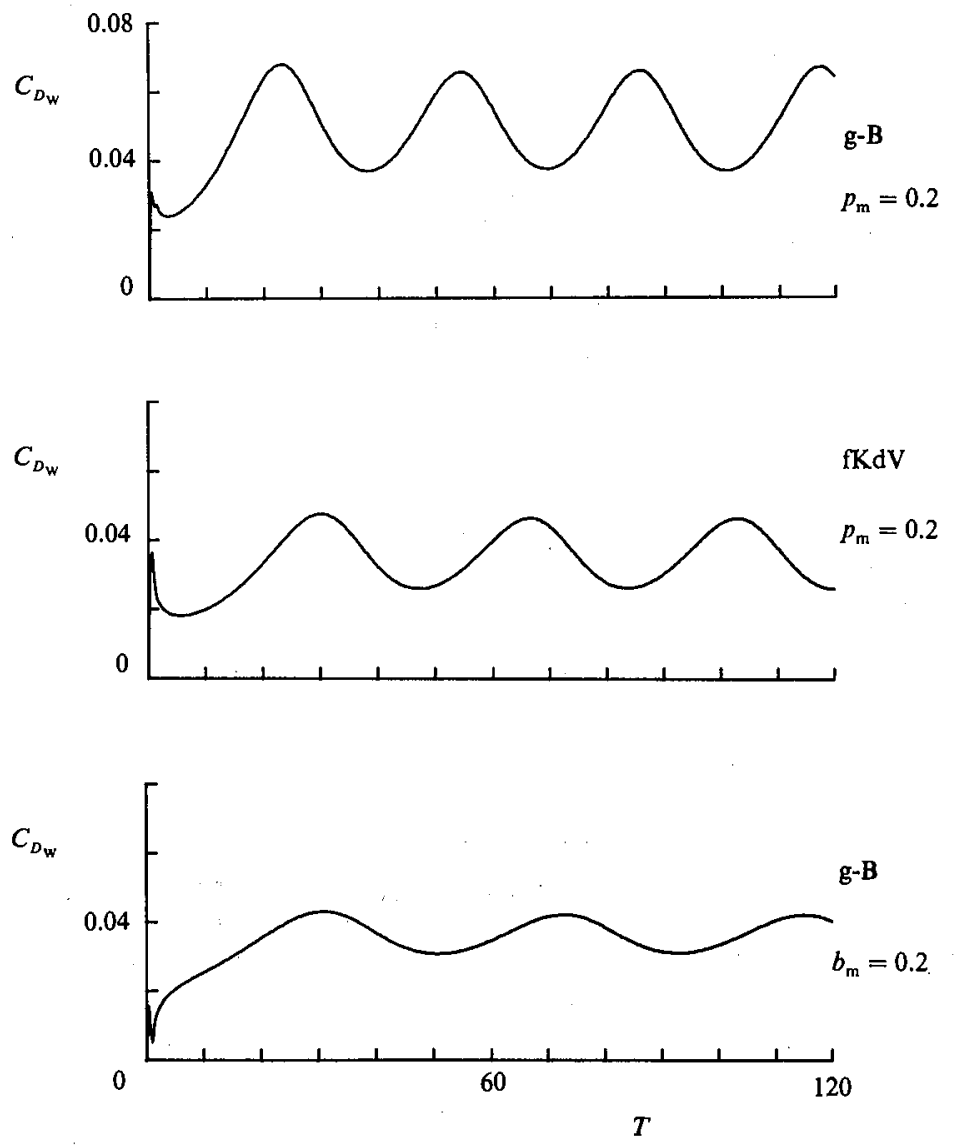

FIGURE 7. Comparison of $(a)$ the surface wave elevation $\zeta$ at $T=120$ and $(b)$ the wave resistance coefficient $C_{D_{\mathrm{w}}}$ predicted by the g-B and fKdV models with surface pressure or bottom topography given by (35). All cases are for $L / h_{0}=2.0, F=1.0$ and $p_{\mathrm{m}}$ or $b_{\mathrm{m}}=0.2$. 


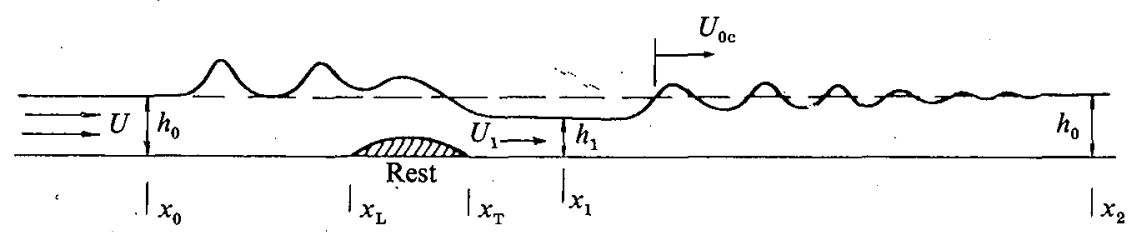

FIGURE 8. Illustration of the transcritical water-wave problem in a frame fixed with respect to the disturbance which is originally moving with uniform velocity $U$ to the left.

left at $x_{0}$. The second is the uniform state with depth $h_{1}$ and fluid velocity $U_{1}$ at $x_{1}$ in the region of depressed water just behind the disturbance, and the third is the freestream state recovered further downstream of the trailing wavetrain at $x_{2}$. From a balance of mass and energy between these uniform states of the flow, Wu (1987) developed formulas to predict the wave amplitudes $a_{\mathrm{s}}$, the period of generation $T_{\mathrm{s}}$, the depth $h_{1}$ of depression, and the mean wave drag on a steadily moving disturbance $\bar{C}_{D_{\mathrm{W}}}$, once any one of these quantities is given. Here, we extend Wu's results, which were developed only for the critical case of Froude number 1.0, to cover all transcritical speeds. Similar mass and energy integrals were developed by Grimshaw \& Smyth (1986); however, the present work considers in addition the wave resistance, the depressed supercritical region following the disturbance, as well as the receding velocity of the trailing wavetrain.

In the body frame, $x=X+F T$ and $t=T$, where the coordinate $X$ and time $T$ are the same as those used above for the fluid frame, the fKdV equation (20) becomes

$$
\zeta_{t}+\left(F-1-\frac{3}{2} \zeta\right) \zeta_{x}-\frac{1}{6} \zeta_{x x x}=\frac{1}{2}\left(p_{\mathrm{a}}+b\right)_{x}
$$

Integrating this equation between the two uniform states, $x_{0}$ to $x_{1}$, and taking the time average over the period $T_{\mathrm{s}}$ of a precursor solitary wave generation gives

$$
\frac{m_{\mathrm{s}}}{T_{\mathrm{s}}}=\frac{3}{4}\left(1-h_{1}\right)^{2}+(F-1)\left(1-h_{1}\right)
$$

where $m_{\mathrm{s}}=4\left(a_{\mathrm{s}} / 3\right)^{\frac{1}{2}}$ is the excess mass of fluid above the undisturbed level corresponding to a single free solitary wave of amplitude $a_{\mathrm{s}}$, and $h_{1}$ is the depth of water in the depression region at $x_{1}$. Similarly, with the integration of (39) ending at the leading edge of the disturbance $x_{\mathrm{L}}$, we obtain

$$
\frac{m_{\mathrm{S}}}{T_{\mathrm{s}}}=\frac{3}{4}\left(\bar{\zeta}_{\mathrm{L}}\right)^{2}-(F-1) \overline{\zeta_{\mathrm{L}}}
$$

where as in $\mathrm{Wu}(1987)$ the oscillatory part of $\zeta_{\mathrm{L}}(t)$ and the surface curvature are assumed small compared with the average wave height at $x_{L}$, denoted by $\bar{\zeta}_{\mathrm{L}}$. Although the upstream-advancing waves, upon refined observation, are slowly evolving and vary slightly from free solitary waves (Lee 1985), they are taken for the present consideration as fully developed and as free solitary waves. From (40) and (41) $\bar{\zeta}_{\mathrm{L}}$ can be found as

$$
\bar{\zeta}_{\mathrm{L}}=1-h_{1}+\frac{4}{3}(F-1) .
$$

Energy integrals obtained from multiplying (39) by $\zeta$ and integrating it from $x_{0}$ to $x_{1}$ and from $x_{0}$ to $x_{\mathrm{L}}$ give, respectively,

$$
\begin{aligned}
& \frac{E_{\mathrm{s}}}{T_{\mathrm{s}}}=\bar{C}_{D_{\mathrm{W}}}-\left(1-h_{1}\right)^{3}-(F-1)\left(1-h_{1}\right)^{2}, \\
& \frac{E_{\mathrm{s}}}{T_{\mathrm{s}}}=\left(\bar{\zeta}_{\mathrm{L}}\right)^{3}-(F-1)\left(\bar{\zeta}_{\mathrm{L}}\right)^{2},
\end{aligned}
$$




\begin{tabular}{|c|c|c|c|c|c|c|c|c|c|c|c|}
\hline$F$ & $\beta$ & $a_{\mathrm{s}}$ & $T_{\mathrm{s}}$ & $\bar{C}_{D_{\mathrm{W}}}$ & $F_{1}$ & $F_{0 \mathrm{e}}$ & $a_{\mathrm{s}}$ & $T_{\mathrm{s}}$ & $\bar{C}_{D_{\mathrm{W}}}$ & $F_{1}^{\prime}$ & $F_{0 \mathrm{c}}$ \\
\hline 0.85 & 0.269 & 0.137 & 45.3 & 0.0121 & 1.137 & 0.848 & 0.112 & 55.6 & 0.00965 & 1.144 & 0.795 \\
\hline 0.90 & 0.249 & 0.201 & 47.3 & 0.0119 & 1.165 & 0.868 & 0.200 & 47.9 & 0.0121 & 1.170 & 0.811 \\
\hline 0.95 & 0.214 & 0.274 & 50.7 & 0.0118 & 1.175 & 0.876 & 0.272 & 50.9 & 0.0118 & 1.179 & 0.838 \\
\hline 1.00 & 0.185 & 0.352 & 56.0 & 0.0111 & 1.194 & 0.908 & 0.370 & 54.7 & 0.0127 & 1.196 & 0.860 \\
\hline 1.05 & 0.150 & 0.444 & 66.0 & 0.0107 & 1.206 & 0.930 & 0.481 & 65.7 & 0.0123 & 1.207 & 0.887 \\
\hline 1.10 & 0.109 & 0.538 & 88.0 & 0.00873 & 1.212 & 0.916 & 0.633 & 92.7 & 0.0108 & 1.212 & 0.918 \\
\hline & & from & $\mathrm{ame}$ & al integ & ion 0 & (39) & & fron & quatio & $45)-(49)$ & \\
\hline
\end{tabular}

TABLE 1. Comparison of results from numerical integration of (39) and prediction from equations $(45)-(49)$

where $E_{\mathrm{s}}=8\left(a_{\mathrm{s}} / 3\right)^{\frac{3}{2}}$ is the total energy associated with a free solitary wave of amplitude $a_{\mathrm{s}}$, and $\bar{C}_{D_{\mathrm{W}}}$ is the mean wave resistance acting on the disturbance (22). Using (42), $\bar{\zeta}_{\mathrm{L}}$ can be eliminated from (43) and (44), which can be rearranged along with (40) as

$$
\begin{gathered}
a_{\mathrm{s}}=2(\beta+4 \delta)(\beta+\delta) / \beta, \\
T_{\mathrm{s}}=\frac{16}{3}\left(\frac{2(\beta+\delta)}{3 \beta^{3}(\beta+4 \delta)}\right)^{\frac{1}{2}}, \\
\bar{C}_{D_{\mathrm{W}}}=2(\beta+2 \delta)^{3},
\end{gathered}
$$

where $\beta=1-h_{1}$ and $\delta=\frac{1}{3}(F-1)$.

Thus, for a given disturbance moving at constant transcritical speed (given $\delta$ ), we need only observe the water depth in the depleted region (observed $\beta$ ), then we can predict the amplitude of the upstream-advancing waves, the generation period and wave resistance using formulas (45)-(47). Table 1 shows that these simple formulas predict the period of generation to within about $5 \%$ of that obtained from the numerical computation for $F$ greater than 0.85 ; the numerical results in this case are for the fKdV equation with forcing given by (35) with $p_{\mathrm{m}}=0.1$ and $L / h_{0}=2.0$. Similar agreement is obtained for the upstream wave amplitude and wave resistance at the lower Froude numbers; however, at supercritical speeds both $a_{\mathrm{s}}$ and $\bar{C}_{D_{\mathrm{w}}}$ predicted by (45) and (47) appear to overestimate the numerical results (figure 9).

From the conservation of mass between $x_{0}$ and $x_{1}, U h_{0}-U_{1} h_{1}=m_{\mathrm{s}} / T_{\mathrm{s}}$, we obtain the Froude number $F_{1}=U_{1} / c_{0}$ of the flow in the depressed region behind the topography as

$$
F_{1}=F+\frac{\beta}{(1-\beta)}\left(1-\frac{3}{4} \beta\right) .
$$

By considering mass and momentum conservation in a frame fixed relative to the first zero-crossing of the trailing wavetrain (Lamb 1932, §187), we obtain

$$
F_{0 \mathrm{c}}=\left(U-U_{0 \mathrm{c}}\right) / c_{0}=\left[\frac{1}{2} h_{1}\left(1+h_{1}\right)\right]^{\frac{1}{2}},
$$

where $U_{0 \mathrm{c}}$ is the velocity of the zero-crossing relative to the moving disturbance, and $F_{0 \mathrm{c}}$ is the Froude number of the leading edge of the trailing wave system referenced to the frame with no flow at $x= \pm \infty$. Table 1 shows that the values of $F_{1}$ given by (48) are very close to the values obtained from the numerical calculations, and that $F_{1}$ is always supercritical and varies only by a few percent within this transcritical range. On the other hand, $F_{0 c}$ is always subcritical and approaches 1.0 as the 


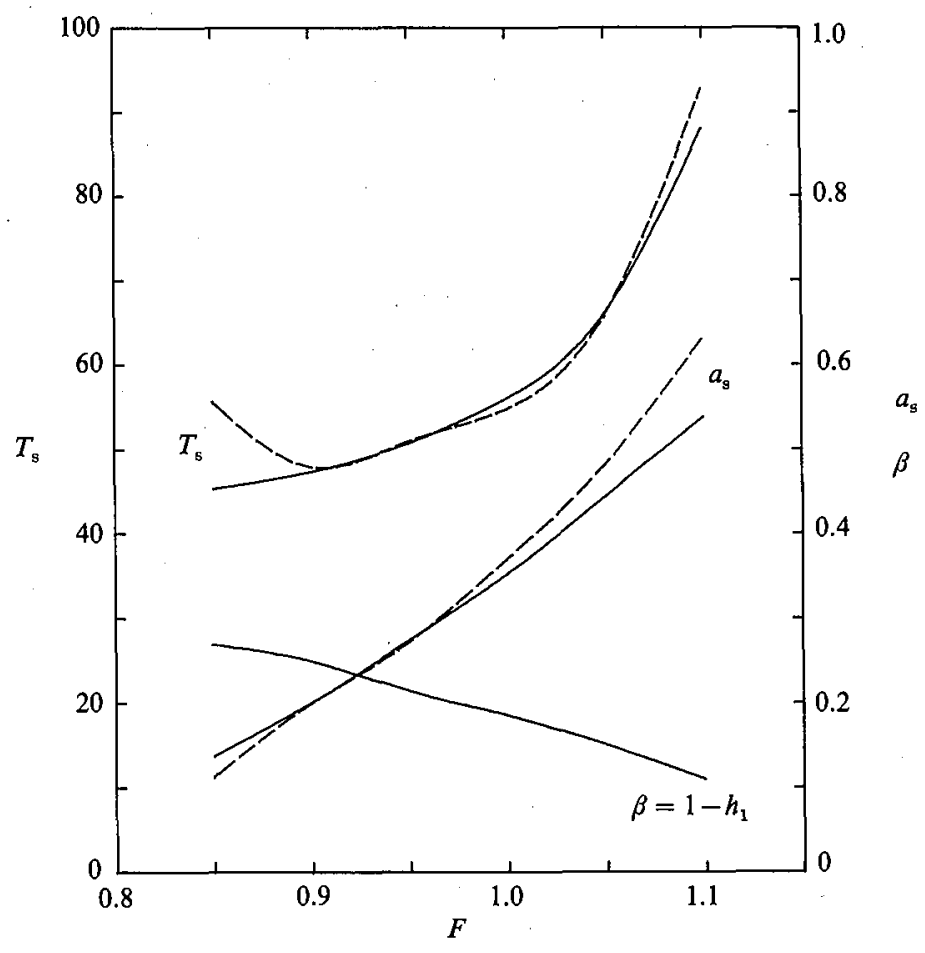

FIgURe 9. Comparison of the precursor solitary wave generation period $T_{\mathrm{s}}$ and the averaged amplitude of the first few generated solitary waves $a_{8}$, predicted from the mass-energy theorem (dashed curves), and numerical results (solid curves) for the fKdV model. Also shown is the numerical result for $\beta=1-h_{1}$, which was taken as input data along with $\delta=\frac{1}{3}(F-1)$ for the mass-energy theorem, equation (45) and (46). Numerical results are obtained from the fKdV model for the pressure distribution given by (35) with $p_{\mathrm{m}}=0.1$ and $L / h_{0}=2.0$.

disturbance speed approaches the limiting supercritical value beyond which upstream waves cease to occur $(F \approx 1.2)$. Furthermore, the estimates of $F_{0 \mathrm{c}},(49)$, are within about $7 \%$ of the numerical results for all transcritical speeds.

\section{Experimental results and comparison with models}

With the objectives to observe and explore experimentally the fascinating phenomenon of the generation of nonlinear waves due to continuous forcing, to evaluate the numerical models versus experimental observations, and to focus directions for further investigations of the underlying mechanism, we carried out a series of experiments using a two-dimensional topographical disturbance which was made to move uniformly along the bottom of a shallow water tank. The bottom topography was preferred to the surface pressure for its ease of construction and implementation. Efforts were directed toward accurate measurements of the wave elevation and, since the phenomenon is unsteady, we positioned several wave gauges at various locations in the tank to monitor the evolution of the waves during and after their generation.

Experiments were conducted in an open flume $7.5 \mathrm{~m}$ long, $0.75 \mathrm{~m}$ wide and $0.6 \mathrm{~m}$ deep and water depths varied from 4.0 to $5.33 \mathrm{~cm}$. The sidewalls and bottom of the flume were constructed of plate glass and the bottom unevenness was measured at 
less than $\pm 0.1 \mathrm{~cm}$. A two-dimensional topography with a chord of $L=4.9 \mathrm{~cm}$, height of $0.65 \mathrm{~cm}$ at its midchord, and span of $72 \mathrm{~cm}$ was machined of aluminium. In cross-section the bump had a circular arc top and a flat base. It was positioned just above the floor of the flume with a gap of $0.05 \mathrm{~cm}$ at the highest location of the floor, and was rigidly fastened to a towing carriage by two thin vertical bars adjacent to the channel inner side walls. The carriage ran on ball-bearing wheels along two parallel tracks mounted above the flanged top of the channel sidewalls. Wave elevations were measured using parallel-wire resistance-type wave gauges and the speed of the carriage was measured by a tachometer in contact with the track.

One set of experimental data was obtained from 2 runs of the disturbance along the flume. Each run was made at the same speed but in opposite directions. Three wave gauges were fixed relative to the flume, and were mounted on a support beam, which spanned the entire length of the tank. In this way we obtained wave gauge records at 5 fixed locations from 2 runs of the experiment. Since one of the fixed wave gauges was positioned at the middle of the measuring interval, it was used to check the repeatability of the 2 runs. Another wave gauge was mounted on the carriage and moved with the disturbance. The position of the moving wave gauge was $1.0 \mathrm{~L}$ upstream (in one run) or $5.0 \mathrm{~L}$ downstream (in the return passage) from the leading edge of the bump. Thus each set of data consisted of 5 fixed and 2 moving wave gauge records and a carriage speed curve, all of which were recorded as functions of time. To eliminate any sidewalls effects the wave elevations were measured at points as close as possible to the longitudinal centreplane of the tank. Since the position of the bump is a key to the phase of the wave system, we used the experimentally obtained carriage speed record at the initial acceleration stage as an input to the numerical simulation.

The signals from the wave gauges and tachometer were digitized by an analog-todigital $(\mathrm{A} / \mathrm{D})$ data acquisition system with 12-bit resolution and a sampling rate of $20 \mathrm{~Hz}$ per channel. A Sanborn thermal chart recorder (Model 358-100A) along with a Sanborn low-gain amplifier bank (Model 958-2900) were used for immediate visual inspection of the data. An analog back-up of the data was simultaneously recorded on a Hewlett-Packard FM tape recorder (Model 3968A).

Calibration of wave gauges was done before and after the experiments for each water depth. The wave gauges responded linearly to changes in water surface displacement and a proportional conversion constant was obtained by using the least-square fit. The average of two calibrations was used for the experimental data obtained between two calibrations. The calibration range was typically $\pm 2 \mathrm{~cm}$, and the relative error between the two calibrations was typically $2-3 \%$. The error range can be estimated as about $5 \%$ for the highest waves measured in the experiments.

To interpret more accurately the comparison between the experimental data and the numerical results, we estimated the effects of energy dissipation on solitons due to bottom friction. The empirical formula suggested by Daily \& Stephan (1952) for the attenuation of a solitary wave in a channel with smooth sidewalls and bottom was used to estimate that a soliton would attenuate less than $5 \%$ at the end of our experiments. Thus the effects of viscosity on wave propagation were not corrected for in the comparison between experimental data and numerical computations.

The presence of a viscous boundary layer around the bump may be thought to increase the effective strength of the bump for the following reasons. As a rough estimate of the viscous effects, we can use the displacement thickness of a flat plate in steady laminar flow (typical Reynolds number is $3.5 \times 10^{4}$ ) as an effective increment of the bump height, which was given in dimensional form as $\delta_{1}=$ 
$1.72(v x / U)^{\frac{1}{2}}$ (see Schlichting 1979). Takin, $x=4.9 \mathrm{~cm}$ and $U=40 \mathrm{~cm} / \mathrm{s}$, we obtain $\delta_{1}=0.06 \mathrm{~cm}$. Thus, the displacement thickness near the trailing edge is about the same as the clearance between the bump and the channel floor. The clearance, however, develops Couette flow, which will in turn induce some back-pressure gradient and impedance to the flow through the clearance. The bottom unevenness was $\pm 0.1^{\prime} \mathrm{cm}$, which is not negligible compared with the bump height, $0.65 \mathrm{~cm}$. For the numerical simulations, we assumed that the bottom was flat at its mean position and that there was no flow between the base of the bump and the channel floor. In an attempt to account for bottom unevenness, the bottom-bump clearance, and presence of a viscous boundary layer, an effective bump height of $0.8 \mathrm{~cm}$ was used instead of the actual bump height of $0.65 \mathrm{~cm}$. In terms of this effective bump height, experiments were performed for $b_{\mathrm{m}} / h_{0}$ ranging from 0.15 to 0.2 .

All the main features indicated earlier, namely the upstream-advancing solitary waves, the elongating depressed region, and the trailing wavetrain, were observed in the experiments. Experimental data along with the numerical solutions predicted by the $\mathrm{fKdV}$ and the g-B model are shown in figure 10 for $b_{\mathrm{m}}=0.15,0.2$ and for $F=0.7,0.8,0.9,1.0,1.1$. We selected the smallest and the largest disturbances for comparison to accentuate the differences between theory and experiment, and more extensive measurements are given by Lee (1985).

From this comparison we find that the fKdV model slightly overpredicts the amplitude and number of precursor solitary waves experimentally observed for subcritical speeds, gives good agreement at critical speeds for small disturbances, and underpredicts the upstream waves at supercritical speeds. For the trailing waves the $\mathrm{fKdV}$ model predicts their amplitudes well at critical and supercritical speeds, but overpredicts the experiments at subcritical speeds. The tendency for the fKdV to overestimate the experiments becomes more pronounced for larger disturbances.

The g-B model slightly underpredicts the amplitude and number of upstreamadvancing solitary waves experimentally observed and overpredicts the amplitude of the trailing waves for the entire range of Froude numbers and disturbance strengths tested. Thus, the $\mathrm{g}-\mathrm{B}$ model shows a more consistent agreement with variations in $F$ and $b_{\mathrm{m}}$ than the fKdV model. Further, this is consistent with the ordering of the flow quantities assumed in the derivation of the respective models and the requirements made for the fKdV model that $F$ be near 1 .

The results of these comparisons with experiments are considered good for both models except for the trailing waves at high subcritical speeds (about $0.8<F<0.9$ ) and for precursor solitary waves at low supercritical speeds (about $1.1<F<1.2$ ), where variance between the numerical and experimental data are most pronounced, especially for relatively strong disturbances. It is precisely in these cases that wave breaking was observed during the experiments.

Because the experiments also reflect other physical effects including viscosity, bottom unevenness, and bottom-bump clearance, it is difficult to say definitely which model performs better in an overall manner. Over the range of the parameters spanned in the current study, the differences between the results predicted by the two models are about the same as those between the experimental data and the results predicted by either of the two models. The $\mathrm{g}-\mathrm{B}$ model has the advantage that it gives more consistent results over the whole range of Froude numbers and disturbance strengths. The $\mathrm{fKdV}$ model is no less competent in its performance near $F \approx 1$, and has the advantage that it may be more suitable for future analytical treatment as illustrated in $\$ 5$.

We mention here several other observations of qualitative nature which we noted 


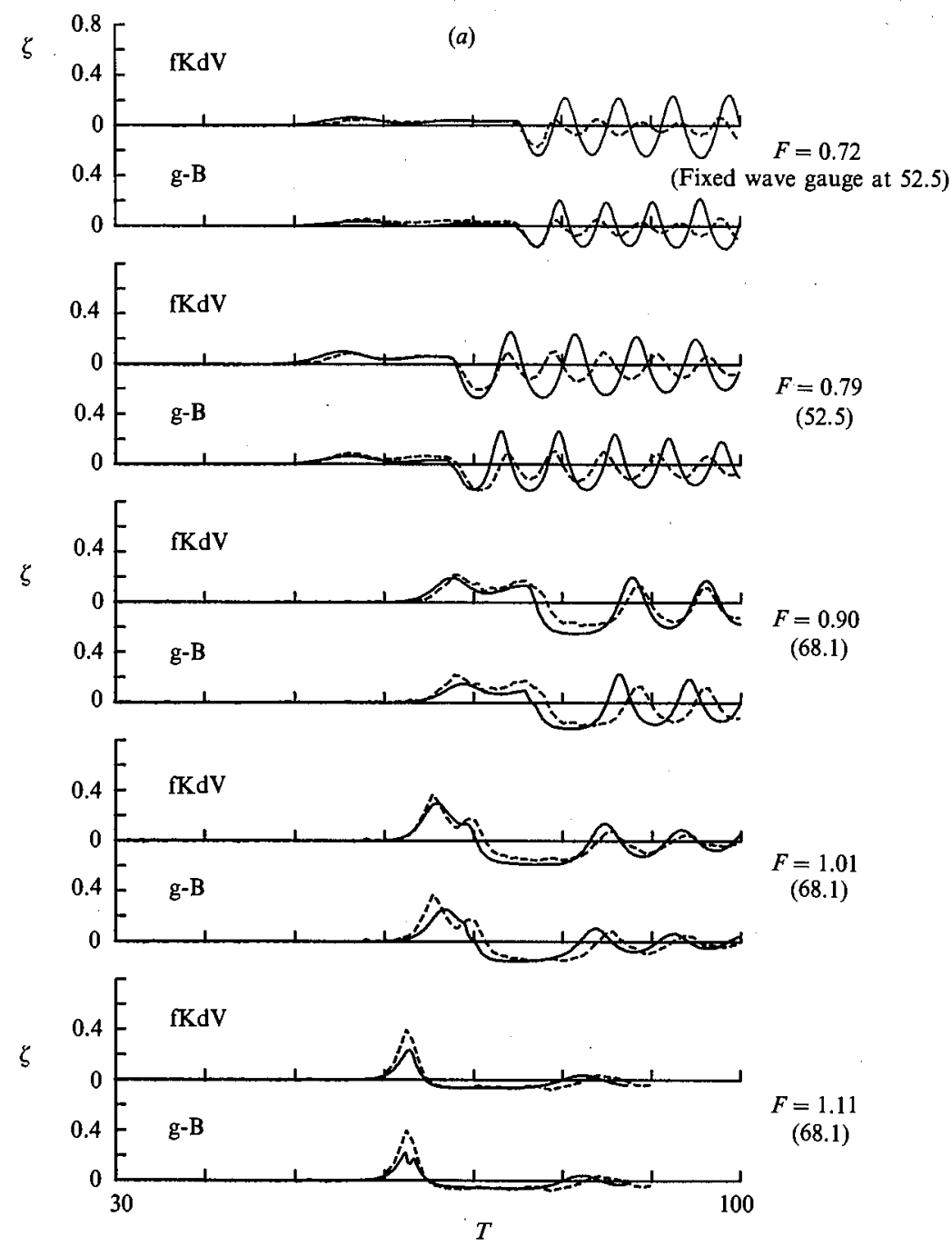

Figure $10(a)$. For caption see facing page.

while undertaking the experiments and which may reflect on the nature of this new phenomenon. (i) We often observed that when a sufficiently strong upstreamadvancing wave was formed, the wave first emerged clean near the disturbance, and then, as this wave propagated upstream, it began to break some time after it left the disturbance region. This supports the contention that these upstream waves are still evolving even as they move ahead of the disturbance. (ii) A series of tests were conducted to determine if the vertical position of the disturbance in the water column would influence the resulting wave development. Although no quantitative experimental data are given here, we can report that all the related data appeared to be quite independent of the disturbance position. Furthermore, Lee (1987) showed that, within the approximation of the $\mathrm{KdV}$ model, a surface pressure, a submerged body and a bottom topography, with identical functional distribution, all act equivalently.

Owing to the limited length of the tank it is difficult to confirm the supposition 


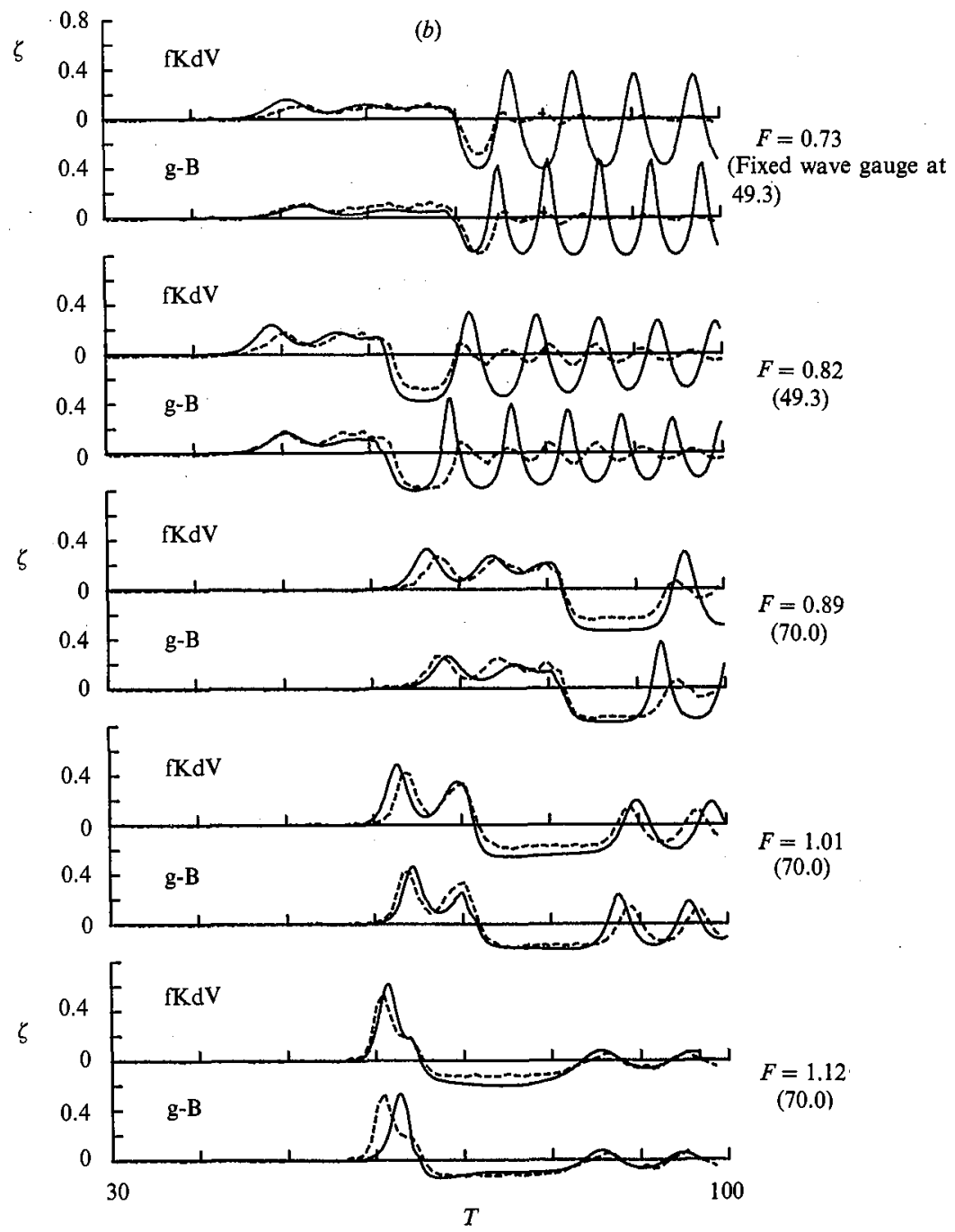

Frgure 10. Comparison between experimental data (dashed curves) and the numerical results of the $\mathrm{fKdV}$ and g-B models (solid curves) for various transcritical Froude numbers and for $(a) b_{\mathrm{m}}=$ $0.15, L / h_{0}=0.92$, and $(b) b_{\mathrm{m}}=0.20, L / h_{0}=1.23$. All data are taken from fixed wave gauges located at the distances indicated in parentheses (given in units of $h_{0}$ ) upstream of the starting position of the disturbance.

that these runaway solitons will be physically generated without end for $F \geqslant 1$. However, based on the overall agreement between the experimental data and the numerical results, we may conclude that the generation of the runaway solitons for $F \geqslant 1$ will last for a very long time, at least over the range of parameters used in these experiments. For $F<1$, whether a limiting state may be approached in finite time remains to be seen.

This work was jointly sponsored by ONR Contract N00014-82-K-0443, NR 062737, and NSF Grant MSM-8706045. The computations were performed on a VAX $11 / 780$ in the Booth computing center of Caltech and on a CRAY X-MP/48 at the San Diego Supercomputer Center, and at the National Center for Supercomputing 
Applications (University of Illinois at Urbana-Champaign). The donation of graphics equipment by Tektronix is gratefully acknowledged. The assistance of Michelle Teng in performing some of the numerical computations is also appreciated.

\section{REFERENCES}

Akylas, T. R. 1984 On the excitation of long nonlinear water waves by a moving pressure distribution. J. Fluid Mech. 141, 455-466.

Benjamin, T. B., Bona, J. L. \& Mahony, J. J. 1972 Model equations for long waves in nonlinear dispersive systems. Phil. Trans. R. Soc. Lond. A 272, 47-78.

Bullough, R. K. \& Caudrey, P.J. 1980 Solitons. In Topics in Current Physics, Vol. 17. Springer.

Cole, S. L. 1985 Transient waves produced by flow past a bump. Wave Motion 7, 579-587.

DaIly, J. W. \& Stephan, S. C. 1952 The solitary wave-its celerity, profile, internal velocities and amplitude attenuation. Hydro. Lab., Dept. Civil Engr., Massachusetts Institute of Technology Tech. Rep. 8.

Dodd, R. K., Embeck, J. C., Gibbon, J. D. \& Morris, H. S. 1982 Solitons and Nonlinear Wave Equations. Academic.

Ertekin, R. C. 1984 Soliton generation by moving disturbances in shallow water: Theory, computation and experiments. Ph.D. thesis, University of Calif., Berkeley, CA.

Ertekin, R. C., Webster, W. C. \& Wehausen, J. V. 1984 Ship-generated solitons. In Proc. 15th Symp. on Naval Hydrodynamics, pp. 347-364. Washington, DC: National Academy Press.

Ertekin, R. C., Webster, W. C. \& Wehausen, J. V. 1986 Waves caused by a moving disturbance in a shallow channel of finite width. J. Fluid Mech. 169, 275-292.

Green, A. E. \& NAGHDI, P. M. $1976 a$ A derivation of equations for wave propagation in water of variable depth. J. Fluid Mech. 78, 237-246.

Green, A. E. \& NaGHdi, P. M. $1976 b$ Directed fluid sheets. Proc. R. Soc. Lond. A 347, $447-473$.

Grimshaw, R. H. J. \& Smyth, N. F. 1986 Resonant flow of a stratified fluid over topography. J. Fluid Mech. 169, 429-464.

Katsis, C. \& AkyLaS, T. R. 1987 On the excitation of long nonlinear water waves by a moving pressure distribution. Part 2. Three-dimensional effects J. Fluid Mech. 177, 49-65.

LAMB, H. 1932 Hydrodynamics. Cambridge University Press.

LEe, S. J. 1985 Generation of long water waves by moving disturbances. Ph.D. thesis, California Institute of Technology, Pasadena, CA.

LEE, S. J. 1987 Generation of long water waves by moving submerged bodies. J. Soc. Naval Arch. Korea 24-2, 55-61.

Lepelletier, T. G. 1981 Tsunamis-harbor oscillations induced by nonlinear transient long waves. Ph.D. thesis, California Institute of Technology, Pasadena, CA.

Lepelletier, T. G. \& RaIchlen, F. 1987 Harbour oscillations induced by nonlinear transient long waves. ASCE J. Waterway, Port, Coastal and Ocean Engng 113-4, 381-400.

MEI, C. C. 1986 Radiation of solitons by slender bodies advancing in a shallow channel. J. Fluid Mech. 162, 53-67.

Melville, W. K. \& Helfrich, K. R. 1987 Transcritical two-layer flow over topography. J. Fluid Mech. 178, 31-52.

Miles, J. W. 1980 Solitary waves. Ann. Rev. Fluid Mech. 12, 11-43.

Rebit, C. \& Soltani, G. 1984 Solitons and Particles. World Scientific.

SCHEMBer, H. R. 1982 A new model for three-dimensional nonlinear dispersive long waves. Ph.D. thesis, California Institute of Technology, Pasadena, CA.

Schlichting, H. 1979 Boundary Layer Theory, 7th edn. McGraw-Hill.

SHIELdS, J. J. 1986 A direct theory for waves approaching a beach. Ph.D. thesis, University of California at Berkeley, Berkeley, CA.

Sмутн, N. F. 1987 Modulation theory solution for resonant flow over topography. Proc. R. Soc. Lond. A 409, 79-97. 
Wнiтнам, G. B. 1974 Linear and Nonlinear Waves. Wiley.

Wu, D. M. \& WU, T. Y. 1982 Three-dimensional nonlinear long waves due to moving surface pressure. In Proc. 14th Symp. on Naval Hydrodynamics, pp. 103-125. Washington, DC: National Academy Press.

Wu, D. M. \& WU, T. Y. 1987 Precursor solitons generated by three-dimensional disturbances moving in a channel. In Non-linear Water Waves. IUTAM Symposium, Tokyo, Japan 1987 (ed. K. Horikawa \& H. Maruo), pp. 69-75. Springer.

WU, T.Y. 1979 On tsunamis propagation-evaluation of existing models. In TsunamisProceedings of the National Science Foundation Workshop (ed. L. S. Hwang \& Y. K. Lee), pp. 110-149. Pasadena, CA: Tetra Tech. Inc.

Wu, T. Y. 1981 Long waves in ocean and coastal waters. J. Eng. Mech. Div. ASCE 107, $501-522$.

WU, T. Y. 1987 Generation of upstream-advancing solitons by moving disturbances. J. Fluid Mech. 184, 75-99.

ZaBusky, N. J. \& Kruskat, M. D. 1965 Interaction of 'solitons' in a collisionless plasma and the recurrence of initial states. Phys. Rev. Lett. 15, 240-243.

ZHU, J. 1986 Internal solitons generated by moving disturbances. Ph.D. thesis, California Institute of Technology, Pasadena, CA.

ZHU, J., WU, T. Y. \& YATES, G. T. 1986 Generation of internal runaway solitons by moving disturbances. In Proc. 16th Symp. on Naval Hydrodynamics (ed. W. C. Webster), pp. 186-198. Washington, DC: National Academy Press.

ZHU, J., WU, T. Y. \& YATES, G. T. 1987 Internal solitary waves generated by moving disturbances. In Stratified Flows (Third Intl Symp. on Stratified Flows; Feb. 3-5, 1987, Pasadena, CA) (ed. E. J. List \& G. H. Jirka). D. Reidel (in press). 